\title{
Mean Curvature Evolution of Entire Graphs
}

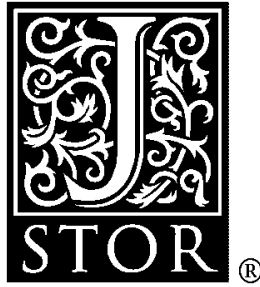

\author{
Klaus Ecker; Gerhard Huisken
}

The Annals of Mathematics, 2nd Ser., Vol. 130, No. 3. (Nov., 1989), pp. 453-471.

Stable URL:

http://links.jstor.org/sici?sici=0003-486X\%28198911\%292\%3A130\%3A3\%3C453\%3AMCEOEG\%3E2.0.CO\%3B2-D

The Annals of Mathematics is currently published by Annals of Mathematics.

Your use of the JSTOR archive indicates your acceptance of JSTOR's Terms and Conditions of Use, available at

http://www.jstor.org/about/terms.html. JSTOR's Terms and Conditions of Use provides, in part, that unless you have obtained prior permission, you may not download an entire issue of a journal or multiple copies of articles, and you may use content in the JSTOR archive only for your personal, non-commercial use.

Please contact the publisher regarding any further use of this work. Publisher contact information may be obtained at http://www.jstor.org/journals/annals.html.

Each copy of any part of a JSTOR transmission must contain the same copyright notice that appears on the screen or printed page of such transmission.

The JSTOR Archive is a trusted digital repository providing for long-term preservation and access to leading academic journals and scholarly literature from around the world. The Archive is supported by libraries, scholarly societies, publishers, and foundations. It is an initiative of JSTOR, a not-for-profit organization with a mission to help the scholarly community take advantage of advances in technology. For more information regarding JSTOR, please contact support@jstor.org. 


\title{
Mean curvature evolution of entire graphs
}

\author{
By Klaus Ecker and Gerhard Huisken
}

We consider immersions

$$
\text { F: } M^{n} \rightarrow \mathbf{R}^{n+1}
$$

of $n$-dimensional hypersurfaces in $\mathbf{R}^{n+1}$. We say that $M^{n}$ moves by mean curvature if there is a one-parameter family $\mathbf{F}_{t}=\mathbf{F}(\cdot, t)$ of immersions with corresponding images $M_{t}=F_{t}(M)$ such that

$$
\begin{aligned}
\frac{d}{d t} \mathbf{F}(p, t) & =\mathbf{H}(p, t), \quad p \in M, \\
\mathbf{F}(p, 0) & =\mathbf{F}_{0}(p)
\end{aligned}
$$

is satisfied for some initial data $\mathbf{F}_{0}$. Here $\mathbf{H}(p, t)$ is the mean curvature vector of the hypersurface $M_{t}$ at $\mathbf{F}(p, t)$.

Mean curvature flow was first studied by Brakke [1], in the context of geometric measure theory. Smooth compact surfaces moving by their mean curvature were investigated in [5], [6].

Here we shall assume that $M$ can be written as an entire graph; i.e. there exists a vector $\omega \in \mathbf{R}^{n+1},|\omega|=1$, such that for a choice of unit normal $\boldsymbol{v}$ for $M$ we have

$$
\langle v, \omega\rangle>0
$$

everywhere on $M$. Then the system

$$
\left(\frac{d}{d t} \mathbf{F}(p, t)\right)^{\perp}=\mathbf{H}(p, t)
$$

which up to tangential diffeomorphisms is equivalent to (1), corresponds to the quasilinear equation

$$
\frac{d}{d t} u=\sqrt{1+|D u|^{2}} \operatorname{div}\left(\frac{D u}{\sqrt{1+|D u|^{2}}}\right) .
$$

Here $\perp$ denotes the normal component of a vector, $u$ is the graph representation for $M_{t}$ with respect to the hyperplane defined by $\omega$ and $D$ indicates 
differentiation in this hyperplane. In the graphical setting it is therefore possible to deal with problem (1) entirely from the viewpoint of partial differential equations. However, due to the geometric nature of equation (1) it turns out to be much more convenient to derive estimates directly from calculations on the hypersurfaces; see Sections 2, 3 and 4 below.

Our first result says that any polynomial growth rate for the height and the gradient of the initial surface $M_{0}$ is preserved during the evolution. We then show in Section 4 that in the case of Lipschitz initial data with linear growth, equation (1) has a smooth solution for all times. This result follows from a priori estimates for the curvature and higher derivatives of the curvature on $M_{t}$, which are global in space direction and interior in time direction. In Section 5 we study the asymptotic behaviour of these solutions as $t \rightarrow \infty$. We prove that our family of surfaces asymptotically approaches a selfsimilar solution of (1), provided the initial graph was "straight" at infinity in the sense that for some $\delta>0, C<\infty$,

$$
|\langle\mathbf{F}, \boldsymbol{v}\rangle| \leq C(1+|\mathbf{F}|)^{1-\delta} .
$$

More precisely, we show that after appropriate rescaling, the surfaces converge to a solution of the equation

$$
\mathbf{F}^{\perp}=\mathbf{H}
$$

which characterizes expanding selfsimilar solutions of (1). This result should be compared with Theorem 3.5 in [6], where it was shown that singularities of the mean curvature flow behave asymptotically like contracting selfsimilar solutions of (1), characterized by the equation

$$
\mathbf{F}^{\perp}=-\mathbf{H} .
$$

While equation (4) has many non-trivial solutions, we show in the appendix that the only entire graphs satisfying equation (5) are planes.

Finally we prove in Section 6 that condition (3) is indeed necessary for asymptotic convergence. We give an example of a slowly oscillating initial surface violating (3) which does not converge asymptotically.

\section{The monotonicity formula}

In the following we shall not distinguish between the image $\mathbf{F}(p, t)$ of a point $p \in M$ and its coordinate vector $\mathbf{x}=\mathbf{x}(p, t)$. For a fixed point $\left(\mathrm{x}_{0}, t_{0}\right) \in$ $\mathbf{R}^{n+1}$ we define the "backward heat kernel" $\rho=\rho(\mathbf{x}, t)$ by

$$
\rho(\mathbf{x}, t)=\left(4 \pi\left(t_{0}-t\right)\right)^{-n / 2} \exp \left(\frac{-\left|\mathbf{x}_{0}-\mathbf{x}\right|^{2}}{4\left(t_{0}-t\right)}\right), \quad t_{0}>t,
$$


such that

$$
\frac{d}{d t} \rho=-\Delta \rho+\rho\left(\frac{\left\langle\mathbf{x}_{0}-\mathbf{x}, \mathbf{H}\right\rangle}{\left(t_{0}-t\right)}-\frac{1}{4} \frac{\left|\left(\mathbf{x}_{0}-\mathbf{x}\right)^{\perp}\right|^{2}}{\left(t_{0}-t\right)^{2}}\right) .
$$

Here and in the following, $\Delta=\Delta_{t}$ denotes the Laplace-Beltrami operator on $M_{t}$. It was shown in [6], Theorem 3.1, that this implies the monotonicity formula

$$
\frac{d}{d t} \int_{M_{t}} \rho d \mu_{t}=-\int_{M_{t}} \rho\left|\mathbf{H}+\frac{1}{2 \tau}\left(\mathbf{x}-\mathbf{x}_{0}\right)^{\perp}\right|^{2} d \mu_{t}
$$

where $d \mu_{t}$ is the measure on $M_{t}$ and $\tau=t_{0}-t$. Proceeding as in [6] we obtain more generally for a function $f=f(\mathrm{x}, t)$ on $M$ that

$$
\frac{d}{d t} \int_{M} f \rho d \mu_{t}=\int_{M}\left(\frac{d}{d t} f-\Delta f\right) \rho d \mu_{t}-\int_{M} f \rho\left|\mathbf{H}+\frac{1}{2 \tau}\left(\mathbf{x}-\mathbf{x}_{0}\right)^{\perp}\right|^{2} d \mu_{t}
$$

All integrals are finite and integration by parts is permitted for the surfaces and functions we are going to consider in the sequel.

1.1 Corollary. Suppose the function $f=f(x, t)$ satisfies the inequality

$$
\left(\frac{d}{d t}-\Delta\right) f \leq \mathbf{a} \cdot \nabla f
$$

for some vector field a, where $\nabla$ denotes the tangential gradient on $M$. If $a_{0}=\sup _{M \times\left[0, t_{1}\right]}|\mathbf{a}|<\infty$ for some $t_{1}>0$, then

$$
\sup _{M_{t}} f \leq \sup _{M_{0}} f
$$

for all $t \in\left[0, t_{1}\right]$.

Proof. Let $k=\sup _{M_{0}} f$ and define $f_{k}=\max (f-k, 0)$. Then we derive from (8)

$$
\left(\frac{d}{d t}-\Delta\right) f_{k}^{2} \leq 2 f_{k} \mathbf{a} \cdot \nabla f_{k}-2\left|\nabla f_{k}\right|^{2} .
$$

Using Young's inequality we obtain, in the weak sense, that

$$
\left(\frac{d}{d t}-\Delta\right) f_{k}^{2} \leq \frac{1}{2} a_{0}^{2} f_{k}^{2}
$$

We may now employ (7) with $f_{k}^{2}$ instead of $f$ and choose $t_{0}>t, \mathbf{x}_{0}$ arbitrary in the definition of $\rho$ to conclude

$$
\frac{d}{d t} \int f_{k}^{2} \rho d \mu_{t} \leq \frac{1}{2} a_{0}^{2} \int f_{k}^{2} \rho d \mu_{t}
$$

which yields the desired result. 


\section{A priori height estimates}

We define the height of $M$ with respect to the hyperplane orthogonal to $\omega$ by

$$
u=\langle\mathbf{x}, \omega\rangle
$$

and observe from (1) that

$$
\left(\frac{d}{d t}-\Delta\right) u=0
$$

In terms of a local orthonormal frame $\left\{\mathbf{e}_{i}\right\}_{1 \leq i \leq n}$ on $M$ we then have the formula

$$
\nabla \boldsymbol{u}=\left\langle\mathbf{e}_{i}, \boldsymbol{\omega}\right\rangle \mathbf{e}_{i} .
$$

Notice that the function $\approx$ mentioned in the introduction denotes the height of $M$ over a fixed point $\hat{\mathbf{x}}$ in the hyperplane, whereas $u(p, t)$ denotes the height of $F(p, t)$ for a fixed $p \in M$. It immediately follows from (9) and Corollary 1.1 that $u$ stays uniformly bounded, if it is uniformly bounded at time $t=0$. To deal with the case of polynomial growth, we need a technical lemma.

2.1 Lemma i). The function $\eta_{1}=\eta_{1}(\mathbf{x}, t)$ defined by

$$
\eta_{1}(\mathbf{x}, t)=|\mathbf{x}|^{2}+2 n t
$$

satisfies

$$
\left(\frac{d}{d t}-\Delta\right) \eta_{1}=0
$$

ii) The function $\eta_{2}=\eta_{2}(\mathbf{x}, t)$ defined by

$$
\eta_{2}(\mathbf{x}, t)=1+|\mathbf{x}|^{2}-u^{2}+2 n t
$$

satisfies for arbitrary $p$

$$
\left(\frac{d}{d t}-\Delta\right) \eta_{2}^{p}=-p(p-1)\left|\nabla \eta_{2}\right|^{2} \eta_{2}^{p-2}+2 p \eta_{2}^{p-1}|\nabla u|^{2}
$$

Proof. We have in view of (1)

$$
\frac{d}{d t} \eta_{1}=2\langle\mathbf{x}, \mathbf{H}\rangle+2 n
$$

and the first identity then follows from

$$
\Delta \eta_{1}=\mathbf{e}_{i}\left(2\left\langle\mathbf{x}, \mathbf{e}_{i}\right\rangle\right)=2\langle\mathbf{x}, \mathbf{H}\rangle+2 n .
$$


Since by (9)

$$
\left(\frac{d}{d t}-\Delta\right) u^{2}=-2|\nabla u|^{2}
$$

we have, using (i),

$$
\left(\frac{d}{d t}-\Delta\right) \eta_{2}=2|\nabla u|^{2}
$$

which implies the second identity.

Now let $M_{t}$ be a smooth solution of (1) which grows at most polynomially. We show that $u(\cdot, t)$ satisfies the same polynomial growth estimate as $u(\cdot, 0)$. Note that the nonnegative function $|\mathbf{x}|^{2}-u^{2}$ measures distance in the hyperplane orthogonal to $\omega$.

2.2 Proposition. If for some $c_{0}<\infty, p \geq 0$, the inequality

$$
u^{2} \leq c_{0}\left(1+|\mathbf{x}|^{2}-u^{2}\right)^{p}
$$

is satisfied on $M_{0}$, then for all $t>0$,

$$
u^{2} \leq c_{0}\left(1+|\mathbf{x}|^{2}-u^{2}+(2 n+4(p-1)) t\right)^{p} .
$$

Proof. From Lemma 2.1 we compute for

$$
\eta=\eta(\mathbf{x}, t)=1+|\mathbf{x}|^{2}-u^{2}+(2 n+4(p-1)) t
$$

the evolution equation

$$
\begin{aligned}
\left(\frac{d}{d t}-\Delta\right) u^{2} \eta^{-p}= & -2 \eta^{-p}|\nabla u|^{2}-p(p+1) \eta^{-p-2}|\nabla \eta|^{2} u^{2} \\
& -2 p \eta^{-p-1} u^{2}|\nabla u|^{2}-4(p-1) p \eta^{-p-1} u^{2} \\
& -4 p \eta^{-p-1} u \nabla u \cdot \nabla \eta
\end{aligned}
$$

Using Young's inequality we obtain

$$
\left|4 p \eta^{-p-1} u \nabla u \cdot \nabla \eta\right| \leq 2 \eta^{-p}|\nabla u|^{2}+2 p^{2} u^{2} \eta^{-p-2}|\nabla \eta|^{2} .
$$

Now observe that $\nabla_{i} u=\left\langle\mathbf{e}_{i}, \omega\right\rangle$ implies

$$
\nabla_{i} \eta=2\left\langle\mathbf{e}_{i}, \mathbf{x}-\langle\mathbf{x}, \omega\rangle \omega\right\rangle
$$

which yields

$$
|\nabla \eta|^{2} \leq 4 \eta
$$


Thus we derive

$$
\left(\frac{d}{d t}-\Delta\right) u^{2} \eta^{-p} \leq 0
$$

and the result follows from Corollary 1.1.

\section{A priori gradient estimates}

To ensure that $M_{t}$ stays a graph for all times we have to estimate $\langle v, \omega\rangle$ from below or equivalently

$$
v=\langle v, \omega\rangle^{-1}
$$

from above. Let $A=\left\{h_{i j}\right\}$ be the second fundamental form.

3.1 LeMMA. The quantity $v$ satisfies the evolution equation

$$
\left(\frac{d}{d t}-\Delta\right) v=-|A|^{2} v-2 v^{-1}|\nabla v|^{2} .
$$

Proof. From [5], Lemma 3.3, we know that $(d / d t) v=\nabla H$. Therefore

$$
\frac{d}{d t} v=-v^{2}\langle\nabla H, \omega\rangle
$$

On the other hand

$$
\begin{aligned}
\Delta v & =\mathbf{e}_{i}\left(-v^{2}\left\langle\nabla_{\mathbf{e}_{i}} v, \omega\right\rangle\right)=\mathbf{e}_{i}\left(-v^{2}\left\langle h_{i l} \mathbf{e}_{l}, \omega\right\rangle\right) \\
& =-v^{2}\langle\nabla H, \omega\rangle+v|A|^{2}+2 v^{-1}|\nabla v|^{2}
\end{aligned}
$$

and the conclusion follows.

3.2 Corollary. If $v$ is bounded at time $t=0$, it remains bounded by the same constant.

3.3 Remark. For the equation on $\mathbf{R}^{n}$

$$
\frac{d}{d t} u=\operatorname{div}\left(\frac{D u}{\sqrt{1+|D u|^{2}}}\right)
$$

which describes mean curvature flow in $e_{n+1}$ direction, a corresponding result is obtained in [2] from interior gradient estimates.

We can also derive polynomial estimates for $v$ similar to those derived in Section 2. 
3.4 Proposition. If for some $c_{1}<\infty, p \geq 0$,

$$
v \leq c_{1}\left(1+|\mathbf{x}|^{2}-u^{2}\right)^{p}
$$

at time $t=0$, then for $t>0$ the inequality

$$
v(\mathbf{x}, t) \leq c_{1}\left(1+|\mathbf{x}|^{2}-u^{2}+2 n t\right)^{p}
$$

holds.

Proof. From Lemma 2.1 and Lemma 3.1 we compute

$$
\begin{aligned}
\left(\frac{d}{d t}-\Delta\right) v \eta_{2}^{-p}= & -|A|^{2} v \eta_{2}^{-p}-2 v^{-1}|\nabla v|^{2} \eta_{2}^{-p} \\
& -p(p+1) v\left|\nabla \eta_{2}\right|^{2} \eta_{2}^{-p-2}-2 p v \eta_{2}^{-p-1}|\nabla u|^{2} \\
& -2 p \eta_{2}^{-p-1} \nabla v \cdot \nabla \eta_{2} .
\end{aligned}
$$

We estimate

$$
\left|2 p \eta_{2}^{-p-1} \nabla v \nabla \eta_{2}\right| \leq 2 v^{-1}|\nabla v|^{2} \eta_{2}^{-p}+\frac{1}{2} p^{2} v \eta_{2}^{-p-2}\left|\nabla \eta_{2}\right|^{2}
$$

and the conclusion follows since $p \geq 0$.

\section{Curvature estimates and longtime existence}

From now on we shall only consider the case of linear growth; i.e. we assume that for some fixed constant $c_{1} \geq 1$ the inequality

$$
v \leq c_{1}
$$

holds everywhere on $M_{0}$. Corollary 3.2 then ensures that (10) remains valid for all $t>0$.

To guarantee longtime existence of a solution for the mean curvature flow, it is crucial to obtain a priori bounds for the second fundamental form on $M_{t}$. In Theorem 4.4 we derive uniform estimates for the curvature and all derivatives of the curvature which are interior in time and allow us to prove existence of a longtime smooth solution to the flow for Lipschitz initial data. Notice that these estimates do not follow from the standard quasilinear theory since $u$ may be unbounded.

4.1 LemMa. The curvature satisfies the inequality

$$
\left(\frac{d}{d t}-\Delta\right)|A|^{2} v^{2} \leq-2 v^{-1} \nabla v \cdot \nabla\left(|A|^{2} v^{2}\right) .
$$


Proof. From [5], Corollary 3.5, we have the evolution equation

$$
\begin{aligned}
\left(\frac{d}{d t}-\Delta\right)|A|^{2} & =-2|\nabla A|^{2}+2|A|^{4} \\
& \leq-2|\nabla| A||^{2}+2|A|^{4} .
\end{aligned}
$$

Together with the identity

$$
\left(\frac{d}{d t}-\Delta\right) v^{2}=-2|A|^{2} v^{2}-6|\nabla v|^{2}
$$

derived from Lemma 3.1 this yields

$$
\begin{aligned}
\left(\frac{d}{d t}-\Delta\right)|A|^{2} v^{2} \leq & -2|\nabla| A||^{2} v^{2}-6|\nabla v|^{2}|A|^{2} \\
& -2 \nabla|A|^{2} \cdot \nabla v^{2}
\end{aligned}
$$

Using Young's inequality we then estimate

$$
\begin{aligned}
-2 \nabla|A|^{2} \cdot \nabla v^{2} \leq & -\nabla|A|^{2} \cdot \nabla v^{2}-4 v|A| \nabla|A| \cdot \nabla v \\
= & -v^{-2} \nabla v^{2} \cdot \nabla\left(|A|^{2} v^{2}\right)+v^{-2}\left|\nabla v^{2}\right|^{2}|A|^{2} \\
& -4 v|A| \nabla|A| \cdot \nabla v \\
\leq & -2 v^{-1} \nabla v \cdot \nabla\left(|A|^{2} v^{2}\right)+6|\nabla v|^{2}|A|^{2} \\
& +2|\nabla| A||^{2} v^{2}
\end{aligned}
$$

which implies the result.

4.2 Corollary. If $M_{t}$ is a smooth solution of (1) with bounded gradient and bounded curvature on each $M_{t}$, then there is the a priori estimate

$$
\sup _{M_{t}}|A|^{2} v^{2} \leq \sup _{M_{0}}|A|^{2} v^{2} .
$$

Proof. Notice that

$$
v^{-1}|\nabla v| \leq|A| v
$$

The result then follows from Lemma 4.1 and Corollary 1.1 with $\mathrm{a}=-2 v^{-1} \nabla v$.

Using the uniform estimate on $|A|^{2}$ we can now proceed exactly as in [6], Proposition 2.3, to estimate all derivatives of $A$ in terms of their initial data.

4.3 Proposition. If $M_{t}$ is a smooth solution of (1) such that $v,|A|^{2}$, $|\nabla A|^{2}, \ldots,\left|\nabla^{m} A\right|^{2}$ are bounded on each $M_{t}$, then we have for all $t \geq 0$ the 
a priori estimate

$$
\sup _{M_{t}}\left|\nabla^{m} A\right| \leq C(m)
$$

where $C(m)$ only depends on $m, n, c_{1}$ and $\sup _{M_{0}}\left|\nabla^{j} A\right|$ for $0 \leq j \leq m$.

We now derive estimates interior in time for the curvature and all its derivatives.

4.4 Proposition. Let $M_{t}$ be a smooth solution of (1) satisfying (10). Then for each $m \geq 0$ there is a constant $C(m)$ depending only on $c_{1}, n$ and $m$ such that

$$
t^{m+1}\left|\nabla^{m} A\right|^{2} \leq C(m)
$$

holds uniformly on $M_{t}$.

Proof. To establish the case $m=0$ we compute from Lemma 3.1 and Lemma 4.1

$$
\begin{aligned}
\left(\frac{d}{d t}-\Delta\right)\left(2 t|A|^{2} v^{2}+v^{2}\right) & \leq-2 v^{-1} \nabla v \cdot \nabla\left(2 t|A|^{2} v^{2}\right)-6|\nabla v|^{2} \\
& \leq-2 v^{-1} \nabla v \cdot \nabla\left(2 t|A|^{2} v^{2}+v^{2}\right)
\end{aligned}
$$

Again Corollary 1.1 yields that the estimate

$$
2 t|A|^{2} v^{2}+v^{2} \leq c_{1}^{2}
$$

holds uniformly on $M_{t}$. We now proceed by induction on $m$ in a way similar to that in [6], Proposition 2.3. From [5], Theorem 7.1 we have for arbitrary $l \geq 0$ the inequality

$$
\begin{aligned}
\left(\frac{d}{d t}-\Delta\right)\left(t^{l+1}\left|\nabla^{l} A\right|^{2}\right) \leq & -2 t^{l+1}\left|\nabla^{l+1} A\right|^{2}+(l+1) t^{l}\left|\nabla^{l} A\right|^{2} \\
& +C(l, n) t^{l+1} \sum_{i+j+k=l}\left|\nabla^{i} A\right|\left|\nabla^{j} A\right|\left|\nabla^{k} A\right|\left|\nabla^{l} A\right| .
\end{aligned}
$$

Suppose (11) is established up to $(m-1)$. Then we estimate

$$
\begin{aligned}
t^{l+1} & \sum_{i+j+k=l}\left|\nabla^{i} A\right|\left|\nabla^{j} A\right|\left|\nabla^{k} A\right|\left|\nabla^{l} A\right| \\
\leq & C t^{l+1} \sum_{i+j+k=l} t^{-i / 2-j / 2-1}\left|\nabla^{k} A\right|\left|\nabla^{l} A\right| \leq C t^{l / 2} \sum_{k \leq l} t^{k / 2}\left|\nabla^{k} A\right|\left|\nabla^{l} A\right| \\
\leq & C \sum_{k \leq l} t^{k}\left|\nabla^{k} A\right|^{2}
\end{aligned}
$$

with constants $C$ depending only on $l, n$ and $c_{1}$. Thus we obtain for all $l \leq m$ 
the inequality

$$
\left(\frac{d}{d t}-\Delta\right)\left(t^{l+1}\left|\nabla^{l} A\right|^{2}\right) \leq-2 t^{l+1}\left|\nabla^{l+1} A\right|^{2}+C \sum_{k \leq l} t^{k}\left|\nabla^{k} A\right|^{2} .
$$

In a first step we may then choose $k_{1}$ so large that

$$
\left(\frac{d}{d t}-\Delta\right)\left(t^{m+1}\left|\nabla^{m} A\right|^{2}+k_{1} t^{m}\left|\nabla^{m-1} A\right|^{2}\right) \leq C \sum_{k \leq m-1} t^{k}\left|\nabla^{k} A\right|^{2} .
$$

We can then successively select $k_{2}, k_{3}, \ldots, k_{m+1}$ such that finally

$$
\begin{aligned}
\left(\frac{d}{d t}-\Delta\right)\left(t^{m+1}\left|\nabla^{m} A\right|^{2}+k_{1} t^{m}\left|\nabla^{m-1} A\right|^{2}+k_{2} t^{m-1}\left|\nabla^{m-2} A\right|^{2}\right. & \\
& \left.+\cdots+k_{m} t|A|^{2}+k_{m+1} v^{2}\right) \leq 0 .
\end{aligned}
$$

Here we used again the evolution equation for $v^{2}$ and the fact that $v \geq 1$. Proposition 4.4 then follows from Corollary 1.1.

Having obtained decay estimates in $t$ we are now able to show also that initial spatial decay behaviour is maintained during the evolution. We will make use of this fact in Section 6.

4.5 Proposition. Let $M_{t}$ be a smooth solution of (1) satisfying (10) and the additional assumption

$$
\left|\nabla^{m} A\right|^{2}(\mathbf{x} s) \leq c_{2}(m)\left(1+|\mathbf{x}|^{2}\right)^{-m-1}, \quad m \geq 0
$$

at time $t=0$. Then for all $t>0$,

$$
\left|\nabla^{m} A\right|^{2} \leq C_{m}\left(1+\left(\sqrt{|\mathbf{x}|^{2}+2 n t}-\sqrt{\beta t}\right)^{2}\right)^{-m-1}
$$

where $\beta=\beta\left(c_{1}\right)>0$ and $C_{m}=C_{m}\left(n, m, c_{1}, c_{2}(0), \ldots, c_{2}(m)\right)$.

Proof. Let us prove (13) for $m=0,1$ only. From there we can proceed by induction in a way similar to that in the proof of Proposition 4.4.

Let $g=|A|^{2} v^{2} \eta+L v^{2}$ where $\eta$ is an arbitrary nonnegative function and $L>0$ will be determined later. Using Lemma 4.1 we compute as in the proof of Proposition 4.4 for the case $m=0$ :

$$
\begin{aligned}
\left(\frac{d}{d t}-\Delta\right) g \leq & \mathbf{b} \cdot \nabla g+2 L \eta^{-2}|\nabla \eta|^{2} v^{2} \\
& +\left(c_{1} t^{-1 / 2}|\nabla \eta|+2 \eta^{-1}|\nabla \eta|^{2}+\left(\frac{d}{d t}-\Delta\right) \eta-2 L\right)|A|^{2} v^{2}
\end{aligned}
$$

where $\mathbf{b}=-2\left(v^{-1} \nabla v+\eta^{-1} \nabla \eta\right)$. Note that we estimated the vector $\mathbf{a}=$ 
$-2 v^{-1} \nabla v$ from Lemma 4.1 using the inequality $v^{-1}|\nabla v| \leq|A| v$, estimate (11) for $m=0$ and the fact that $C(0)=c_{1}^{2} / 2$. We now define

$$
\eta(\mathrm{x}, t)=1+\left(\sqrt{|\mathbf{x}|^{2}+2 n t}-\sqrt{\beta t}\right)^{2}
$$

where $\beta>0$ will be chosen later. We obviously have the inequalities

$$
|\nabla \eta|^{2} \leq 4 \eta
$$

and

$$
|\nabla \eta| \leq 2 \sqrt{|\mathbf{x}|^{2}+2 n t}+2 \sqrt{\beta t}
$$

In view of Lemma 2.1.i), $\eta$ also satisfies

$$
\left(\frac{d}{d t}-\Delta\right) \eta \leq \beta-\sqrt{\frac{\beta}{t}\left(|\mathbf{x}|^{2}+2 n t\right)} .
$$

Therefore we conclude for large enough $\beta=\beta\left(c_{1}\right)$,

$$
\left(\frac{d}{d t}-\Delta\right) g \leq \mathbf{b} \cdot \nabla \mathbf{g}+8 L \eta^{-1} v^{2}+\left(2 c_{1} \beta^{1 / 2}+\beta+8-2 L\right)|A|^{2} v^{2} .
$$

If we now choose $L$ large depending on $\beta$ and $c_{1}$ and define $k=\sup _{M_{0}} g+9 L c_{1}^{2}$, we achieve

$$
\left(\frac{d}{d t}-\Delta\right) g \leq \mathbf{b} \cdot \nabla \mathbf{g}-\eta^{-1}(g-k)
$$

where we also used the estimate $v(x, t) \leq c_{1}$ again.

Let $g_{k}=\max (g-k, 0)$. Since $g_{k} \cdot(g-k)=g_{k}^{2}$, we obtain the result using Corollary 1.1 with $f=g_{k}^{2}$.

In order to prove (13) for $m=1$, we compute as in Proposition 4.4:

$$
\begin{aligned}
\left(\frac{d}{d t}-\Delta\right)|\nabla A|^{2} \eta^{2} & \leq-2\left|\nabla{ }^{2} A\right|^{2} \eta^{2}+c(n)|A|^{2} \eta^{2}|\nabla A|^{2}-2 \nabla \eta^{2} \cdot \nabla|\nabla A|^{2} \\
& \leq c(n)|A|^{2} \eta^{2}|\nabla A|^{2}+8|\nabla \eta|^{2}|\nabla A|^{2} .
\end{aligned}
$$

Since $|\nabla \eta|^{2} \leq 4 \eta$ and $|A|^{2} \eta \leq C_{0}$ (recall that $v \geq 1$ ) we estimate

$$
\left(\frac{d}{d t}-\Delta\right)|\nabla A|^{2} \eta^{2} \leq c\left(n, C_{0}\right)|\nabla A|^{2} \eta \text {. }
$$

Similarly we derive

$$
\left(\frac{d}{d t}-\Delta\right)|A|^{2} \eta \leq-|\nabla A|^{2} \eta+c\left(n, C_{0}\right)|A|^{2} .
$$


Since by Lemma 3.1 and the fact that $v \geq 1$ one has

$$
\left(\frac{d}{d t}-\Delta\right) v^{2} \leq-2|A|^{2}
$$

it is now easy to see that

$$
\left(\frac{d}{d t}-\Delta\right)\left(|\nabla A|^{2} \eta^{2}+K|A|^{2} \eta+L v^{2}\right) \leq 0
$$

for large enough positive constants $K$ and $L$ depending on $n$ and $C_{0}$. The proposition then follows from Corollary 1.1.

Using Proposition 4.4 we can prove existence of a longtime solution for Lipschitz initial data.

4.6 Theonem. If the initial hypersurface $M_{0}$ is Lipschitz continuous and satisfies

$$
\sup _{M_{0}} v \leq c_{1},
$$

then the mean curvature flow problem (1) has a longtime solution which is smooth for all $t>0$ and satisfies the a priori estimates in Corollary 3.2 and Proposition 4.4.

Proof. Suppose first that the initial hypersurface is smooth with $\sup _{M_{0}}\left|\nabla^{m} A\right|$ bounded for all $m \geq 0$. In view of the bound on $v$ the linearization of $\left(2^{\prime}\right)$ is a uniformly parabolic equation and the implicit function theorem guarantees the existence of a unique smooth solution on some short time interval. Our uniform a priori estimates then ensure that this solution extends to all $t>0$. For Lipschitz initial data the result follows by approximation in view of our interior estimates in Proposition 4.4.

\section{Asymptotic behaviour}

In this section we study the behaviour of solutions $M_{t}$ of the mean curvature flow system (1) for large times $t$ in the case of linear growth. For simplicity we shall additionally assume that the initial surface $M_{0}$ has bounded curvature. We saw in Proposition 4.4 that the surfaces $M_{t}$ "flatten out" as $t \rightarrow \infty$, and if they do not diverge to $\infty$ (e.g. if $u$ is bounded), then they must converge to a plane. However, in general the surfaces will move out to infinity at speed proportional to $t^{-1 / 2}$ such that Proposition 4.4 does not yield any information about their global shape.

To study the global shape of $M_{t}$ for $t \rightarrow \infty$ we will now rescale the surfaces in such a way that they do not diverge to infinity and at the same time retain a bound on their curvature. 
Similarly, as in [6], we define

$$
\tilde{\mathbf{F}}(s)=\frac{1}{\sqrt{2 t+1}} \mathbf{F}(t)
$$

where the new time variable $s$ is given by $s=\frac{1}{2} \log (2 t+1), 0 \leq s<\infty$. The normalized equation then becomes

$$
\frac{d}{d s} \tilde{\mathbf{F}}=\tilde{\mathbf{H}}-\tilde{\mathbf{F}}
$$

and the estimates from Proposition 2.2, Corollary 3.2, Corollary 4.2 and Proposition 4.4 translate to

$$
\begin{aligned}
\tilde{u}^{2}(\tilde{\mathbf{x}}, s) & \leq \tilde{c}_{0}\left(1+|\tilde{\mathbf{x}}|^{2}-\tilde{u}^{2}(\tilde{\mathbf{x}}, s)\right), \\
\tilde{v}(\tilde{\mathbf{x}}, s) & \leq c_{1}, \\
|\tilde{A}|^{2}(\tilde{\mathbf{x}}, s) & \leq c_{2},
\end{aligned}
$$

with constants depending only on the initial bounds for the respective quantities on $M_{0}$.

For the rescaled surfaces $\tilde{M}_{s}=\tilde{\mathbf{F}}(\cdot, s)(M)$ we then establish the following result concerning asymptotic convergence.

5.1 Theonem. Suppose $M_{0}$ satisfies the linear growth condition (10) and has bounded curvature. If in addition the estimate

$$
\langle\mathbf{F}, v\rangle^{2} \leq c_{3}\left(1+|\mathbf{F}|^{2}\right)^{1-\delta}
$$

is valid on $M_{0}$ for some constants $c_{3}<\infty, \delta>0$, then the solution $\tilde{M}_{s}$ of the normalized equation (14) converges for $s \rightarrow \infty$ to a limiting surface $\tilde{M}_{\infty}$ satisfying the equation

$$
\mathbf{F}^{\perp}=\mathbf{H}
$$

We will see in the last section that condition (3) is indeed necessary.

5.2 Remarks. i) The result follows from the estimate

$$
\sup _{\tilde{M}_{s}} \frac{(\tilde{H}+\langle\tilde{\mathbf{x}}, \tilde{v}\rangle)^{2} \tilde{v}^{2}}{\left(1+\alpha|\tilde{\mathbf{x}}|^{2}\right)^{1-\varepsilon}} \leq e^{-\beta s} \sup _{M_{0}} \frac{(H+\langle\mathbf{x}, v\rangle)^{2} v^{2}}{\left(1+\alpha|\mathbf{x}|^{2}\right)^{1-\varepsilon}}
$$

which we derive for all $\varepsilon<\delta$ with some constants $\alpha>0, \beta>0$ depending only on $\varepsilon, n, c_{1}$ and $c_{2}$. This implies, in particular, exponentially fast convergence on compact subsets, a result much stronger than the corresponding result in [6] concerning asymptotic behaviour of singularities. 
ii) In view of the interior estimates in Proposition 4.4 the conclusion of Theorem 5.1 remains valid for Lipschitz initial data provided condition (3) is satisfied for some $t_{0}>0$.

iii) Any initial surface $M_{0}$ given by $\mathbf{F}_{0}: M^{n} \rightarrow \mathbf{R}^{n+1}$ satisfying (4) gives rise to an expanding selfsimilar solution of the mean curvature flow in the sense that

$$
\mathbf{F}(t)=\sqrt{2 t+1} \mathbf{F}_{0}
$$

satisfies

$$
\left(\frac{d}{d t} \mathbf{F}\right)^{\perp}=\mathbf{H} .
$$

Theorem 5.1 therefore says that $M_{t}$ becomes asymptotically selfsimilar. Nontrivial examples of surfaces satisfying (4) can be constructed in arbitrary dimensions by choosing for instance a rotationally symmetric cone $\mathscr{C}$ in $\mathbf{R}^{n+1}$ as initial surface for the evolution. From the maximum principle applied to the evolution equation for $H$ we obtain a family of surfaces lying above $\mathscr{C}$ for all times. Thus the limiting hypersurface $M_{\mathscr{G}}$ for the rescaled flow obtained from Theorem 5.1 is nonlinear. Moreover, from Proposition 4.5 one can see that $M_{\mathscr{C}}$ satisfies the estimate

$$
|\langle\mathbf{x}, v\rangle|=|H| \leq \frac{c}{1+|\mathbf{x}|}
$$

and is therefore strongly asymptotic to $\mathscr{C}$.

In the one-dimensional case an example for "curves of constant shape" evolving from a corner was numerically obtained by Brakke in [1], Fig. 3. It is an open problem to understand and possibly classify solutions of equation (4). We show in the appendix that the equation (5) characterizing contracting selfsimilar solutions of the mean curvature flow has only trivial solutions in the class of entire graphs.

We begin the proof of the theorem with the following lemma.

5.2 Lemma. The quantity $\langle\mathbf{x}, \boldsymbol{v}\rangle$ satisfies the evolution equation

$$
\left(\frac{d}{d t}-\Delta\right)\langle\mathbf{x}, \boldsymbol{v}\rangle=|A|^{2}\langle\mathbf{x}, v\rangle-2 H \text {. }
$$

Proof. From the equation $(d / d t) v=\nabla H$ we compute

$$
\frac{d}{d t}\langle\mathbf{x}, \boldsymbol{v}\rangle=-H+\langle\mathbf{x}, \nabla H\rangle
$$


while

$$
\begin{aligned}
\Delta\langle\mathbf{x}, \boldsymbol{v}\rangle & =\mathbf{e}_{i}\left\langle\mathbf{x}, \nabla_{e_{i}} \boldsymbol{v}\right\rangle=\mathbf{e}_{i}\left\langle\mathbf{x}, h_{i k} \mathbf{e}_{k}\right\rangle \\
& =H+\left\langle\mathbf{x}, \nabla_{\mathbf{e}_{i}} \mathbf{e}_{k}\right\rangle h_{i k}+\left\langle\mathbf{x}, \mathbf{e}_{k}\right\rangle \nabla_{k} H \\
& =H-|A|^{2}\langle\mathbf{x}, \boldsymbol{v}\rangle+\langle\mathbf{x}, \nabla H\rangle .
\end{aligned}
$$

We can now show that up to a time-dependent factor condition (3) is preserved for all $s>0$.

5.4 Lemma. Suppose $M_{0}$ satisfies the assumptions of Theorem 5.1. Then on $\tilde{M}_{s}$ we have the estimate

$$
\langle\tilde{\mathbf{x}}, \tilde{\boldsymbol{v}}\rangle^{2} \leq C(s)\left(1+|\tilde{\mathbf{x}}|^{2}\right)^{1-\delta}
$$

with a constant depending on $s$ and $c_{2}$.

Proof. Since the constant in the estimate is allowed to depend on time it is sufficient to look at the unnormalized flow. From Lemma 5.3 we infer

$$
\begin{aligned}
\left(\frac{d}{d t}-\Delta\right)\langle\mathbf{x}, v\rangle^{2} & =2\langle\mathbf{x}, \boldsymbol{v}\rangle^{2}|A|^{2}-4 H\langle\mathbf{x}, \boldsymbol{v}\rangle-2|\nabla\langle\mathbf{x}, \boldsymbol{v}\rangle|^{2} \\
& \leq C\left(\langle\mathbf{x}, \boldsymbol{v}\rangle^{2}+1\right)-2|\nabla\langle\mathbf{x}, \boldsymbol{v}\rangle|^{2}
\end{aligned}
$$

where from now on we denote all constants depending only on $c_{2}$ and $s$ by $C$. We now write $f=\langle\mathbf{x}, v\rangle$, multiply the above equation by a test function $\rho$ and estimate

$$
\left(\frac{d}{d t}-\Delta\right) f^{2} \rho \leq\left(C+2 \rho^{-2}|\nabla \rho|^{2}+\rho^{-1}\left(\frac{d}{d t}-\Delta\right) \rho\right) f^{2} \rho+c \rho .
$$

Choosing $\rho=\eta_{1}^{\delta-1}$ where $\eta_{1}=1+|\mathbf{x}|^{2}+2 n t$ we derive from Lemma 2.1.i), since $0<\delta \leq 1$,

$$
\left(\frac{d}{d t}-\Delta\right) \rho=-(\delta-1)(\delta-2) \eta_{1}^{\delta-3}\left|\nabla \eta_{1}\right|^{2} \leq 0 .
$$

Furthermore,

$$
\rho^{-2}|\nabla \rho|^{2}=(1-\delta)^{2} \eta_{1}^{-2}\left|\nabla \eta_{1}\right|^{2} \leq 4(1-\delta)^{2} \eta_{1}^{-1} \leq 4
$$

since $\left|\nabla \eta_{1}\right|^{2} \leq 4 \eta_{1}$. Altogether we conclude

$$
\left(\frac{d}{d t}-\Delta\right) f^{2} \rho \leq C\left(f^{2} \rho+1\right)
$$

such that by Corollary 1.1, $f^{2} \rho$ can at most grow exponentially in time. This implies the result. 
5.5 Lemma. The normalized quantity $\tilde{H}+\langle\tilde{\mathbf{x}}, \tilde{\mathbf{v}}\rangle$ satisfies the evolution equation

$$
\left(\frac{d}{d s}-\tilde{\Delta}\right)(\tilde{H}+\langle\tilde{\mathbf{x}}, \tilde{\mathbf{v}}\rangle)=\left(|\tilde{A}|^{2}-1\right)(\tilde{H}+\langle\tilde{\mathbf{x}}, \tilde{\boldsymbol{v}}\rangle)
$$

Proof. From [5], Lemma 9.1, and Lemma 5.3 we compute the identities

$$
\begin{aligned}
& \left(\frac{d}{d s}-\Delta\right) \tilde{H}=|\tilde{A}|^{2} \tilde{H}+\tilde{H} \\
& \left(\frac{d}{d s}-\tilde{\Delta}\right)\langle\tilde{\mathbf{x}}, \tilde{\boldsymbol{v}}\rangle=|\tilde{A}|^{2}\langle\tilde{\mathbf{x}}, \tilde{\boldsymbol{v}}\rangle-2 \tilde{H}-\langle\tilde{\mathbf{x}}, \tilde{\boldsymbol{v}}\rangle .
\end{aligned}
$$

This yields (16).

Proof of Theorem 5.1. From (16) we infer

$$
\left(\frac{d}{d s}-\tilde{\Delta}\right)(\tilde{H}+\langle\tilde{\mathbf{x}}, \tilde{\boldsymbol{v}}\rangle)^{2}=2\left(|\tilde{A}|^{2}-1\right)(\tilde{H}+\langle\tilde{\mathbf{x}}, \tilde{\boldsymbol{v}}\rangle)^{2}-2|\nabla(\tilde{H}+\langle\tilde{\mathbf{x}}, \tilde{\boldsymbol{v}}\rangle)|^{2}
$$

The normalized gradient $\tilde{v}$ satisfies the equation

$$
\left(\frac{d}{d s}-\tilde{\Delta}\right) \tilde{v}^{2}=-2|\tilde{A}|^{2} \tilde{v}^{2}-6|\nabla \tilde{v}|^{2} .
$$

We may then proceed exactly as in the proof of Lemma 4.1 to obtain for $f^{2}=(\tilde{H}+\langle\tilde{\mathbf{x}}, \tilde{\boldsymbol{v}}\rangle)^{2} \tilde{v}^{2}$ the inequality

$$
\left(\frac{d}{d s}-\tilde{\Delta}\right) f^{2} \leq-2 f^{2}-2 \tilde{v}^{-1} \nabla \tilde{v} \cdot \nabla f^{2} .
$$

Multiplying by a test function $\rho$ we compute

$$
\begin{aligned}
\left(\frac{d}{d s}-\tilde{\Delta}\right) f^{2} \rho \leq & -2 f^{2} \rho-2 \rho \tilde{v}^{-1} \nabla \tilde{v} \cdot \nabla f^{2} \\
& +f^{2}\left(\frac{d}{d s}-\tilde{\Delta}\right) \rho-2 \nabla \rho \cdot \nabla f^{2} .
\end{aligned}
$$

Now let $0<\varepsilon<\delta$ and define $\rho(\tilde{\mathbf{x}}, s)=\eta_{\alpha}^{\varepsilon-1}(\tilde{\mathbf{x}}) e^{\beta s}$ with $\eta_{\alpha}(\tilde{\mathbf{x}})=1+\alpha|\tilde{\mathbf{x}}|^{2}$ where $\alpha, \beta$ are small positive constants to be determined later. Then the normalized equation (14) implies

$$
\left(\frac{d}{d s}-\tilde{\Delta}\right) \eta_{\alpha}=-2 \alpha\left(|\tilde{\mathbf{x}}|^{2}+n\right)
$$


and therefore

$$
\left(\frac{d}{d s}-\tilde{\Delta}\right) \rho \leq(\beta+2(1-\varepsilon)(\alpha n+1)) \rho .
$$

Moreover,

$$
\begin{aligned}
& -2 \rho \tilde{v}^{-1} \nabla \tilde{v} \cdot \nabla f^{2}-2 \nabla \rho \cdot \nabla f^{2} \\
& =-2\left(\tilde{v}^{-1} \nabla \tilde{v}+\rho^{-1} \nabla \rho\right) \cdot \nabla\left(f^{2} \rho\right) \\
& \quad+2|\nabla \rho|^{2} f^{2} \rho^{-1}+2 f^{2} \tilde{v}^{-1} b \nabla \tilde{v} \cdot \nabla \rho
\end{aligned}
$$

and we obtain from $\left|\nabla \eta_{\alpha}\right|^{2} \leq 4 \alpha \eta_{\alpha}$ the estimate

$$
|\nabla \rho| \leq 2 \alpha^{1 / 2} \rho .
$$

Combining now (17), (18) and (19) and using the fact that $|\nabla \tilde{v}| \leq|\tilde{A}| \tilde{v}^{2}$ we derive for $g=f^{2} \rho$ the inequality

$$
\left(\frac{d}{d s}-\tilde{\Delta}\right) g \leq \mathbf{a} \cdot \nabla \mathbf{g}+\left(\beta+c \alpha^{1 / 2}-2 \varepsilon\right) \mathbf{g}
$$

where $\mathbf{a}=-2\left(\tilde{v}^{-1} \nabla \tilde{v}+\tilde{\boldsymbol{\rho}}^{-1} \nabla \tilde{\boldsymbol{\rho}}\right)$ and $c$ depends on $c_{1}, c_{2}$ and $n$. Choosing then $\alpha, \beta$ suitably small depending on $\varepsilon$ and $c$ we see that

$$
\left(\frac{d}{d s}-\tilde{\Delta}\right) \mathbf{g} \leq \mathbf{a} \cdot \nabla \mathbf{g}
$$

for all $s \geq 0$. Lemma 5.4 ensures that $g$ vanishes at infinity which enables us to apply the parabolic maximum principle to conclude that $g$ is uniformly bounded by its initial data. This proves estimate (15) and completes the proof of Theorem 5.1.

\section{A counterexample}

In this section we want to show that the result of Theorem 5.1 is optimal. The main observation underlying the following arguments is that in case the mean curvature decays at infinity the normalized equation (14) primarily describes a "blow-down" of the initial surface. Hence for initial surfaces which slowly oscillate at infinity and violate condition (3), one would not expect asymptotic convergence as described in Section 5 . We have the following explicit examples.

6.1 Proposition. If $M_{0}$ is the graph of the function

$$
u_{0}(\hat{\mathbf{x}})=u_{0}(|\hat{\mathbf{x}}|)= \begin{cases}|\hat{\mathbf{x}}| \sin \log |\hat{\mathbf{x}}|, & |\hat{\mathbf{x}}| \geq 1 \\ \text { smooth, } & |\hat{\mathbf{x}}| \leq 1,\end{cases}
$$

where $\hat{\mathbf{x}}$ denotes coordinates in $\mathbf{R}^{n}$, then the normalized surfaces $\tilde{M}_{s}$ do not converge to a solution of the limiting equation (4). 
The proposition is a special case of the following lemma from which a large number of further examples can be obtained.

6.2 Lemma. Suppose $M_{0}$ satisfies the assumptions of Proposition 4.5 for $m=0,1$ and suppose there exists a sequence of points $p_{k} \in M^{n}$ such that $\left|\mathbf{x}\left(p_{k}, 0\right)\right| \rightarrow \infty$ and $\left\langle\mathbf{x}\left(p_{k}, 0\right), v\right\rangle^{2}=\gamma\left|\mathbf{x}\left(p_{k}, 0\right)\right|^{2}$ for some $\gamma>0$. Then there exists a sequence of times $s_{k}$ for which $(\tilde{H}+\langle\tilde{\mathbf{x}}, \tilde{v}\rangle)$ does not converge to zero.

Proof. Let us assume for simplicity that $\gamma=1 / 2$. Rescaling estimate (13) we immediately infer that for all $s>0$,

$$
\begin{aligned}
|\tilde{H}|^{2}(\tilde{\mathbf{x}}, s) & \leq \frac{c_{4}}{|\tilde{\mathbf{x}}|^{2}}, \\
|\nabla \tilde{H}|^{2}(\tilde{\mathbf{x}}, s) & \leq \frac{c_{5}}{|\tilde{\mathbf{x}}|^{4}}
\end{aligned}
$$

if $|\tilde{\mathbf{x}}| \geq C=C(n, \beta)$. We now derive by direct calculation from (14)

$$
\left|\frac{d}{d s} \frac{\langle\tilde{\mathbf{x}}, \tilde{\nu}\rangle^{2}}{|\tilde{\mathbf{x}}|^{2}}\right| \leq \frac{c_{6}}{|\tilde{\mathbf{x}}|^{2}}
$$

as well as

$$
\left.|| \tilde{\mathbf{x}}(p, s)\right|^{2}-e^{-2 s}|\tilde{\mathbf{x}}(p, 0)|^{2} \mid \leq c_{7} .
$$

Now let $p_{k}$ be the sequence of points which for all $k \geq 1$ satisfy the relation

$$
\left\langle\mathbf{x}\left(p_{k}, 0\right), v\right\rangle^{2}=\frac{1}{2}\left|\mathbf{x}\left(p_{k}, 0\right)\right|^{2} .
$$

Let us choose a sequence of times $s_{k}$ such that

$$
e^{2 s_{k}}=\varepsilon c_{7}^{-1}\left|\mathbf{x}\left(p_{k}, 0\right)\right|^{2}
$$

with some fixed $0<\varepsilon<\frac{1}{2}$ to be determined. It follows from (20) and (21) that for $s \leq s_{k}$,

$$
\left|\frac{d}{d s} \frac{\left\langle\tilde{\mathbf{x}}\left(p_{k}, s\right), \tilde{\nu}\right\rangle^{2}}{\left|\tilde{\mathbf{x}}\left(p_{k}, s\right)\right|^{2}}\right| \leq 2 \varepsilon c_{6} c_{7}^{-1} e^{2\left(s-s_{k}\right)}
$$

and therefore

$$
\frac{\left\langle\tilde{\mathbf{x}}\left(p_{k}, s_{k}\right), \tilde{\nu}\right\rangle^{2}}{\left|\tilde{\mathbf{x}}\left(p_{k}, s_{k}\right)\right|^{2}} \geq \frac{1}{2}-\varepsilon c_{6} c_{7}^{-1} .
$$

Furthermore we see from (21) that

$$
c_{7}\left(\varepsilon^{-1}-1\right) \leq\left|\tilde{\mathbf{x}}\left(p_{k}, s_{k}\right)\right|^{2} \leq c_{7}\left(\varepsilon^{-1}+1\right) .
$$


In view of the a priori estimate

$$
|\tilde{H}(\tilde{\mathbf{x}}, s)|^{2} \leq \frac{c_{5}}{|\tilde{\mathbf{x}}|^{2}}
$$

for $|\tilde{\mathbf{x}}| \geq c(n, \beta)$, we may choose $\varepsilon>0$ so small that the quantity $(\tilde{H}+\langle\tilde{\mathbf{x}}, \tilde{\boldsymbol{v}}\rangle)^{2}\left(p_{k}, s_{k}\right)$ is uniformly bounded from below. This completes the proof of Lemma 6.2.

\section{Appendix}

We show that in the case of entire graphs the only contracting selfsimilar solutions of the mean curvature flow are hyperplanes.

Proposition. If $M$ is an entire graph of at most polynomial growth satisfying the equation

$$
\boldsymbol{H}=\langle\mathbf{x}, \boldsymbol{v}\rangle,
$$

then $M$ is a plane.

Proof. From (22) we easily compute $\nabla_{i} H=\left\langle\mathbf{x}, \mathbf{e}_{l}\right\rangle h_{i l}$ and hence

$$
\Delta v=|A|^{2} v+2 v^{-1}|\nabla v|^{2}+\left\langle\mathbf{x}, \mathbf{e}_{i}\right\rangle \nabla_{i} v .
$$

We multiply this equation by $\rho=\exp \left(-|x|^{2} / 2\right)$ which after integration by parts leads to

$$
\int_{M}|A|^{2} v \rho d \mu+2 \int_{M} v^{-1}|\nabla v|^{2} \rho d \mu=0,
$$

thus implying the result.

University of Melbourne, Parkville, Australia

Australian National Universtty, Canberra, Australia

\section{REFERENCES}

[1] K.A. Brakke, The Motion of a Surface by its Mean Curvature, Math. Notes, Princeton University Press, Princeton, NJ, 1978.

[2] K. ECKER, Estimates for evolutionary surfaces of prescribed mean curvature, Math. Z. 180 (1982), 179-192.

[3] A. Friedman, Partial Differential Equations of Parabolic Type, Prentice-Hall, Englewood Cliffs, NJ, 1964.

[4] C. Gerhardt, Evolutionary surfaces of prescribed mean curvature, J. Diff. Eq. 36 (1980), 139-172.

[5] G. Huisken, Flow by mean curvature of convex surfaces into spheres, J. Diff. Geom. 20 (1984), 237-266.

[6] Asymptotic behaviour for singularities of the mean curvature flow, to appear in J. Diff. Geom. (1989). 